\title{
EL PROBLEMA MÁS IMPORTANTE QUE TENEMOS
}

\author{
JAMES A. F. STONER \\ Facultad de Negocios Gabelli, Universidad de Fordham \\ Nueva York, Nueva York, EE. UU. \\ stoner@fordham.edu
}

En 1986, Richard Hamming, un científico e innovador intelectual muy respetado, dio un discurso en el Bell Labs Morris Research and Engineering Center en Morristown, N.J., donde recordó de una serie de comidas en ciertos laboratorios, en que comenzaba preguntando: “¿Cuáles son los problemas más importantes de su campo?" Después de una semana les preguntó “¿Qué problemas importantes están abordando ustedes?" Y después de algún tiempo, entró un día y dijo "Si lo que están haciendo no es importante, y piensan que no llevará a algo importante ¿por qué están en los laboratorios Bell trabajando sobre eso?" (Hamming, 1986)

Las preguntas de Hamming no solo se deben plantear a los científicos de uno de los mejores centros de investigación del mundo de todos los tiempos. “¿Cuál es el problema más urgente que enfrentamos hoy en día? ¿Estamos en ello? Y si no, ¿por qué no lo estamos haciendo?"-_on palabras apropiadas para cada uno de nosotros para el poco tiempo que tenemos, afortunados de estar en este planeta tan bonito.

Teniendo en cuenta la historia de los jesuitas y su tradición de "cambiar el mundo" a través de la educación inspirada en innovaciones sociales repetidas (Lowney, 2003), las universidades jesuitas y sus escuelas de negocios están invitadas sobre todo a explorar tales preguntas y descubrir, haciéndolo, las preguntas más importantes que tenemos. Es cierto que el Papa Francisco en Laudato Si' (Francisco, 2015) tiene muy pocas dudas sobre el desafío más urgente que todos, nuestra especie incluso, enfrentamos. Nos pide-en una encíclica muy diferente porque se dirige no solo a los católicos o a los cristianos sino a todos en este planeta-que entablemos un diálogo sobre el rumbo económico, medioambiental, social y cultural, claramente dañino y potencialmente catastrófico en el que nos embarcamos como especie; 
y de ese diálogo, descubrir cómo podemos contribuir a mejorar la trayectoria de nuestras acciones.

Un año después de la publicación de Laudato Si', Paul Krugman, Premio Nobel, también tenía pocas dudas sobre el problema más urgente al que nos enfrentamos, sobre todo si consideramos los asuntos de política global como indicadores de las preocupaciones más importantes de nuestra especie:

\footnotetext{
El año pasado fue el más caliente registrado, por un amplio margen; ello debería (aunque no lo hará) poner fin a las afirmaciones de los negadores del clima de que el calentamiento global ha cesado. La verdad es que el cambio climático sigue asustándonos. Es, con creces, el tema político más importante que enfrentan América y el mundo. (Krugman, 2016)
}

Hace algunos meses, Bill McKibben, uno de los "canarios en la mina de carbón" de la insostenibilidad global desde hace mucho tiempo y también autor de numerosos libros que incluyen The End of Nature (1989) (El fin de la naturaleza) y Eaarth: Making a Life on a Tough New Planet (2010) (Eaarth: Viviendo en un planeta nuevo y duro), hizo esta pregunta alarmante en el subtítulo de su nuevo libro Falter (2019) (Flaquear): “¿Ha empezado el juego humano a desarrollarse hasta el final?”. Jared Diamond, quizás más conocido por su obra Guns, Germs, and Steel: The Fates of Human Societies (1999) (Armas, gérmenes y acero: La sociedad humana y sus destinos) y autor de Collapse: How Societies Choose to Fail or Succeed (2005) (Colapso: Por qué unas sociedades perduran y otras desaparecen), reseñó el libro de McKibben en un artículo en el New York Times Book Review con fecha del 21 de abril, 2019. Notó que

en la primera mitad del libro [McKibben] explica los peligros actuales de la civilización que incluyen el riesgo de una guerra nuclear y también los riesgos múltiples asociados con el cambio climático: el aumento del dióxido de carbono en la atmósfera, las amenazas a la producción de alimentos, el aumento del nivel del mar, el calentamiento del océano y la acidificación.

Luego, Diamond nota lo siguiente:

La parte central del libro habla de las fuerzas opuestas a las soluciones a los problemas planteados en la primera parte-motivados de diversas maneras por el interés propio, las realidades sombrías, el poder, los ideales y las opiniones sobre el papel apropiado del gobierno. Estas fuerzas incluyen Exxon, la pobreza, la desigualdad, Ayn Rand, los hermanos Koch, otros estadounidenses muy ricos, el presidente Trump y Silicon Valley.... 
Finalmente, en la última parte del libro, McKibben ofrece sus motivos de esperanza. Entre los más destacados están los paneles solares, que ofrecen energía renovable barata en todo el mundo, y los movimientos no violentos, cuyos exitosos activistas contra las oposiciones arraigadas y bien armadas han incluido a Gandhi, Martin Luther King Jr., los manifestantes del Día de la Tierra y al propio grupo de McKibben, 350.org.

Diamond concluye su reseña tan rica y valiosa con el párrafo siguiente:

\begin{abstract}
Se requerirán muchas voces diferentes para convencer a los diversos ciudadanos y empresas del mundo a colaborar para resolver los problemas más grandes del mundo. La voz de McKibben ha tenido influencia. Espero que su nuevo libro fortalezca la motivación de los que se solidarizan con su punto de vista. Me temo que no vaya a convencer a los que no los creen. Espero que mi primera predicción sea correcta y la segunda, no. (Diamond, 2019)
\end{abstract}

La semana siguiente, John Lanchester (2019) escribió que el cambio climático "es el mayor desafío al que la humanidad se enfrenta colectivamente" en la primera frase de su reseña en el Times de dos otros nuevos libros (Wallace-Wells, 2019; Rich, 2019).

\title{
¿ENTONCES QUÉ?
}

Al enfrentarse a las realidades de las situaciones recientes, actuales y futuras, los editoriales y artículos del Journal of Management for Global Sustainability nos han invitado a menudo a "afrontar los hechos brutales sin perder la fe," citando a Jim Collins (Collins, 2001) y seguirán haciéndolo, quizás de una manera más fuerte y estridente en el futuro.

Lo aterrador de las perspectivas que sugieren que el cambio climático y la insostenibilidad global son "amenazas existenciales" es que no estamos hablando de las obras de autores como Camus, Kierkegaard y Sartre; estamos hablando de las amenazas a la misma existencia de nuestra propia especie. Una de las principales aportaciones de Laudato Si' es cómo el Papa Francisco declara franca y directamente que la insostenibilidad global es un asunto moral y que los daños que estamos haciendo ahora a los más vulnerables y a las generaciones futuras son errores morales de la mayor magnitud. Las formas que hemos elegido para producir y consumir los bienes y lujos de nuestras vidas y cómo distribuimos los beneficios y costos de esos procesos de producción-consumo-distribución contribuyen al carácter insostenible 
de nuestra situación actual. Todos somos parte del problema de la insostenibilidad global, e incluso las escuelas de negocios también forman parte del problema.

El énfasis claro de Laudato Si' en la inmoralidad de nuestras contribuciones para crear un presente insostenible para muchos y un futuro inhóspito para todos es pertinente al estado actual de la enseñanza, la investigación y en particular, la conceptualización de los miembros de las escuelas de negocios jesuitas. De hecho, incluso si la educación en management de los jesuitas puede ser ligeramente mejor que el promedio cuando se trata de abordar cuestiones de insostenibilidad global, sería difícil defender la afirmación de que la enseñanza dominante en marketing, finanzas y contabilidad en las instituciones jesuitas es muy diferente de la que se encuentra en otras escuelas. Dejando a un lado el curso ocasional en marketing verde o marketing "en la base de la pirámide," sería muy difícil afirmar que los cursos en finanzas, marketing, contabilidad, economía, administración, operaciones, comunicaciones, negociación, leyes y quizás incluso ética en todas las escuelas de negocios, incluidas las jesuitas, no se dedican principalmente a brindarles a nuestros estudiantes las habilidades y actitudes para "tomar-desperdiciar-desperdiciar-rápidoy-más rápido-para-los-ricos-y-más ricos" y sentirse muy bien sobre ellos mismos mientras lo hacen.

Al reconocer que la insostenibilidad global es, en su esencia, un problema moral de la mayor magnitud y no solo un problema empresarial, todas las universidades y sus escuelas de negocios tienen la obligación y la oportunidad de comportarse de manera que les permita dejar de contribuir al problema y empezar a convertirse en medios para encontrar soluciones. La red mundial de escuelas de negocios jesuitas, en particular, está especialmente diseñada para explorar las realidades de nuestra situación actual, reflexionar sobre esas realidades y nuestros recursos para realizar contribuciones positivas y tomar medidas que puedan impactar no solo a las instituciones educativas sino también al mundo.

Arthur Taylor, cuando fue decano de la Escuela de Negocios de Posgrado de la Universidad de Fordham desde finales de los 80 hasta principios de los 90, invitó una vez a Roland Christensen, el experto en la enseñanza de casos en la Escuela de Negocios de Harvard (Harvard Business School o HBS), a participar en un retiro de profesores y dirigir una sesión sobre la enseñanza de casos. A Frank Werner, que 
había sido alumno de Christensen en HBS, se le pidió que hospedara a Christensen durante la visita.

Al final del viaje, Frank le notó a Roland, mientras le conducía al aeropuerto para su vuelo de regreso, que debía de recibir muchas invitaciones de este tipo, y le preguntó por qué había aceptado esta. Christensen respondió que Frank estaba en lo cierto acerca de las invitaciones y que le había preguntado a su decano si debía aceptar esta cuando la recibió. Su decano dijo que sí, que sería bueno aceptarlo porque un área en la que la HBS podría ser competitivamente vulnerable estaba relacionada de alguna manera con el propósito y significado más profundo de las organizaciones empresariales y la educación: un área a la que la espiritualidad y la religión podrían tener un acceso especial. Tenía curiosidad, por lo tanto, por lo que Fordham podría estar haciendo como una escuela de negocios creyente en un área donde la HBS podría ser vulnerable.

Cuando Frank le preguntó a Roland qué había aprendido durante su visita sobre lo que Fordham estaba haciendo en ese dominio, Christensen respondió: "No mucho."

Fordham, desafortunadamente, no estaba aprovechando sus valores y patrimonio jesuitas para promover la transformación de la educación e investigación en management. Está claro que en términos de innovación para la transformación de la educación en management, la respuesta de Christensen probablemente fue cierta para prácticamente todas las escuelas de negocios, las que son creyentes y las que no. Y es probable que todavía sea cierto hoy en día, cuando la necesidad de transformar la educación en management es aún mayor debido a que nuestra situación es mucho más grave.

Tres décadas después de la conversación de Christensen y Werner, la necesidad de transformaciones profundas, impulsadas por la insostenibilidad global, en qué y cómo producimos, distribuimos y consumimos tal como se sugiere en Laudato Si ofrece muchas oportunidades para que las escuelas de negocios jesuitas proporcionen respuestas dramáticamente diferentes para la pregunta que Roland Christensen estaba explorando. Son respuestas que podrían encender el fuego que transformará la educación en management en todo el mundo. 


\section{¿Y AHORA QUÉ? UNA SEGUNDA OPORTUNIDAD PARA LAS ESCUELAS DE NEGOCIOS JESUITAS}

Las escuelas de negocios jesuitas han planteado, de varias maneras muy significativas, enfoques y acciones pioneras que han contribuido activamente y siguen contribuyendo a construir un mundo más justo y sostenible. Los compromisos con la justicia social y la lucha contra la pobreza, por ejemplo, han sido focos importantes en prácticamente toda la educación en management jesuita. Los centros y programas para la sostenibilidad global están ubicados en varios campus, y las escuelas han sido líderes sobresalientes en los dominios de la innovación social y el emprendimiento social. La Asociación Internacional de Escuelas de Negocios Jesuitas (International Association of Jesuit Business Schools o IAJBS) tomó un compromiso profundo con la sostenibilidad global en 2009. Aunque las muchas otras actividades similares son demasiado numerosas para enumerarlas aquí y no deben minimizarse ni pasarse por alto, aún quedan por delante otras oportunidades interesantes que aún no se han aprovechado. Se hablará de tres de ellas a continuación. Si bien son atractivas para las escuelas miembros y los claustros de IAJBS y CJBE (Colleagues in Jesuit Business Education; Colegas en la Educación Empresarial Jesuita) en particular, sin embargo, también presentan oportunidades interesantes para todas las escuelas de negocios. Después de analizar estas tres áreas de oportunidad, proporcionaremos una breve introducción a los artículos en este número de la revista.

\section{EL FORO MUNDIAL DE LA IAJBS}

La IAJBS reconoció esta amenaza existencial hace diez años cuando su $15^{\circ}$ Foro Mundial se reunió en el Xavier Labor Relations Institute (XLRI; Instituto de Relaciones Laborales Javier) en Jamshedpur, Jharkhand, India. Aquel foro mundial, cuyo tema era el liderazgo para la sostenibilidad, era diferente en un grande, y quizás único, aspecto-se presentó una resolución, aprobada por unanimidad y ratificada al día siguiente por la Junta Ejecutiva de la IAJBS. La resolución pidió que el Foro Mundial se dedicara durante los próximos diez años al tema general de contribuir a un mundo más sostenible. Luego, en el Foro Mundial en la Universidad Ateneo de Manila en Manila el año siguiente, Rudy Ang y sus colegas de la IAJBS propusieron que la organización creara una revista sobre la sostenibilidad. El primer número de esa revista, la Journal of Management for Global Sustainability (Revista de Gestión para la Sostenibilidad Global) se publicó en 2013. 
Durante la última década desde 2009, las palabras que se usan para describir el objetivo de lograr un mundo sostenible han evolucionado desde los conceptos de sostenibilidad, desarrollo sostenible, sostenibilidad global y florecimiento. Ahora, quizás, podemos agregar "regeneración," que se refiere no solo a hacer menos daño o ningún daño, sino a restaurar nuestro mundo roto, "cuidar nuestra casa común," como lo diría el Papa Francisco. La definición de sostenibilidad ofrecida en el primer número de esta revista, de manera bastante interesante, abordó explícitamente la necesidad de "curar nuestro mundo roto" sustituyendo las palabras "sin comprometer" tal como se encuentran en la definición popular de desarrollo sostenible de la Comisión Brundtland con la frase "mientras se mejora."

\begin{abstract}
Definimos la sostenibilidad global como ... un proceso que satisface las necesidades de la generación actual al tiempo que mejora la capacidad de las generaciones futuras para satisfacer sus propias necesidades. La sostenibilidad global visualiza un mundo que funciona para todos, sin que nadie se quede fuera. (Stoner, 2013: 2).
\end{abstract}

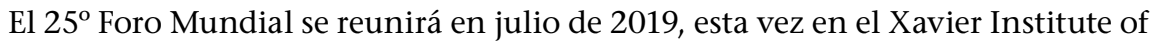
Management (XIMB; Instituto de Gestión Xavier) en Bhubaneswar, India. También servirá como la reunión inaugural de la Sección Regional de Asia del Sur de los Colegas en la Educación Empresarial Jesuita. El tema de este foro, "Innovar y florecer," sigue la definición de sostenibilidad de John Ehrenfeld como "la posibilidad de que la vida humana y de otro tipo florezca en el planeta para siempre" (Ehrenfeld, 2009).

Dado que la reunión de 2019 se cumple en el décimo aniversario del compromiso asumido en la conferencia XLRI en 2009, es muy probable que se ofrezca una nueva resolución invitando al Foro Mundial a comprometerse de nuevo a otros diez años de liderazgo para un mundo sostenible, o tal vez comprometerse con un mundo regenerativo.

El Foro Mundial IAJBS no es, por supuesto, la única conferencia anual que históricamente ha elegido un tema nuevo y diferente cada año. De hecho, casi todas las conferencias lo hacen. Sin embargo, al igual que el Foro Mundial marcó un hito al comprometerse durante diez años con el tema del liderazgo para la sostenibilidad, otras organizaciones profesionales o incluso fundaciones importantes pueden tomar compromisos similares para centrar sus energías y las nuestras en "el mayor desafío de la humanidad." Solo en el ámbito de las organizaciones profesionales para académicos de gestión, por ejemplo la Academy of Management, la Eastern 
Academy of Management y las demás, la conferencia de gestión y de conducta organizativa y otras podrían enviar la señal al mundo de que los problemas del cambio climático e insostenibilidad global ya no deben ser ignorados y que sus miembros van a prestarles la atención que han merecido por mucho tiempo pero que no han recibido. Y tal vez también las principales fundaciones, como la Fundación Bill y Melinda Gates, la Fundación MacArthur, la Fundación Ford, la Fundación Susan Thompson Buffett y otras, harán compromisos similares a largo plazo para reunir los recursos que necesitamos para inspirar, financiar y honrar a aquellos que estén dispuestos y ansiosos por hacer lo que se debe hacer si nosotros y nuestros hijos queremos tener un futuro digno.

\section{NUESTRA TRANSFORMACIÓN Y LA EDUCACIÓN DE NEGOCIOS}

Si buscamos dos transformaciones importantes que nuestra especie pueda necesitar para enfrentar nuestra situación actual de insostenibilidad global, para avanzar hacia un futuro floreciente y regenerativo, una de ellas puede ser a nivel individual y la otra a nivel de sistemas. Como individuos, es posible que emprendamos la "conversión ecológica" que el Papa Francisco pide en Laudato Si'. Es posible que tengamos que convertirnos en personas diferentes como productores, consumidores y ciudadanos. A nivel de sistemas, es posible que tengamos que transformar el sistema defectuoso de producción, distribución, consumo que sirve a gran parte del mundo de manera tan deficiente ya que está destruyendo la capacidad del planeta para sustentar nuestra propia especie y otras.

¿Cómo lograremos estas transformaciones? La red de escuelas de negocios jesuitas podría proporcionar valiosas contribuciones al mundo en estos dos campos.

\section{EXPLORANDO LAS TECNOLOGÍAS PARA UNA CONVERSIÓN ECOLÓGICA}

Hay cierta ironía en el hecho de que estamos invirtiendo miles de millones de dólares para investigar una variedad de tecnologías que aborde los problemas de nuestro sistema productor-distribuidor-consumidor, pero casi nada para explorar cómo podemos convertirnos en el tipo de personas que utilice esas tecnologías para crear un mundo sostenible / floreciente / regenerativo. No estamos haciendo 
inversiones a gran escala para descubrir cómo podemos transformarnos; de hecho, estamos haciendo inversiones en tecnología de punta a nivel de sistemas en un momento en que muchos observadores creen que ya tenemos toda la tecnología que necesitamos para crear un mundo sostenible (p.ej. los 100 proyectos mencionados en la obra de Paul Hawken: Drawdown: The Most Comprehensive Plan Ever Proposed to Reverse Global Warming [Drawdown: el plan más completo jamás propuesto para revertir el calentamiento global (2018)]) y el enfoque integrado para transformar la economía global utilizando las tecnologías existentes como se describe en $A$ Finer Future: Creating an Economy in Service to Life (Un futuro mejor: Creando una economía en servicio a la vida) por Lovins, Wallis, Wijkman y Fullerton (2018). El problema es que no estamos aprovechando las tecnologías que ya tenemos.

Si nos liberamos de pensar automáticamente en la tecnología como algo mecánico, a menudo incorporado en la maquinaria y orientado hacia la producción de productos físicos, y en cambio recordamos que podemos definirla simplemente como "un proceso para hacer algo" o como "un sistema mediante el cual la sociedad proporciona a sus miembros las cosas necesarias o deseadas" (Your dictionary, n.d.), es posible aceptar la invitación a invertir de manera sustancial para descubrir cómo utilizar con mayor eficacia nuestras tecnologías de transformación personal de siglos y milenios existentes, así como descubrir otras nuevas.

Podemos buscar formas para hacer que esas tecnologías de transformación sean más efectivas, rápidas y ligeras para quienes las comparten, las adquieren y las utilizan. Y podemos buscar formas de inventar nuevas tecnologías. Podríamos observar, por ejemplo, que los ejercicios espirituales de San Ignacio, algo cercano a las instituciones jesuitas, pueden considerarse como una tecnología de transformación personal y espiritual de cinco siglos de antigüedad, que se ha mostrado útil repetidamente. La tentación de explorar tecnologías muy prometedoras de transformación personal, por lo tanto, puede ser bastante atractiva dado que muchos miles de millones ya están invirtiendo en la búsqueda de tecnologías a nivel de sistemas destinadas a cambiar nuestras formas de producir, distribuir y consumir.

Cuando nos enfocamos en nosotros mismos y en nuestras formas de estar en el mundo, también es tentador pensar en invertir una cantidad de dinero apreciable en la creación de una serie de centros de investigación innovadores que analizarán las tecnologías de transformación personal. Los Centros de Acción e 
Investigación en Tecnologías Transformacionales (Centers for Action and Research into Transformational Technologies o CARTT) son casi una marca que puede mejorarse enormemente. Sin embargo, independientemente de lo que decidamos mejorar, podría ser valioso observar, a través de la lente de la tecnología, experiencias de transformación sólidas y bien establecidas, como los Ejercicios Espirituales de San Ignacio, el yoga, la meditación, las prácticas de atención plena y la investigación apreciativa, el conjunto de programas e iniciativas de Ashoka U, el conjunto de programas de Landmark Education y muchos otros que brindan un cambio positivo, profundo y duradero en la vida de las personas y en las formas de ser en el mundo. Y luego están surgiendo enfoques que también pueden ser dignos de una exploración seria, como la Teoría U (p. ej., Scharmer, 2016), Gestión humanística (p. ej., Pirson, 2017) y Liderazgo cuántico (Tsao y Laszlo, 2019).

Existe una posibilidad emergente de que uno o más de estos centros puedan comenzar pronto. De hecho, podría ser particularmente atractivo explorar tecnologías de transformación personal en universidades creyentes, tanto jesuitas como de otro tipo, dado que muchas de estas tecnologías centenarias para transformarnos como seres humanos surgen de y / o están basadas en formas espirituales de estar en el mundo. Las universidades creyentes se sentirán en casa ayudando a descubrir qué es lo que hace que estas tecnologías tengan el impacto que tienen, cómo podemos ponerlas a disposición de más pueblos del mundo, cómo podemos hacerlas más baratas, más rápidas, mejores, y muy importante, cómo podemos protegernos de y prevenir su mal uso.

\section{EL LIDERAZGO EN LAS ESCUELAS DE NEGOCIO}

En "Entre dos paradigmas: una lucha por el alma de las escuelas de negocios," Chris Laszlo, Robert Sroufe y Sandra Waddock (Torn Between Two Paradigms: A Struggle for the Soul of Business Schools, 2017) piden actuar para transformar la narrativa neoliberal que domina, en gran medida, la enseñanza de management en el mundo. Como se ha señalado en esta revista y en otras partes, el sistema global de producción, distribución y consumo, que es omnipresente, auto-referente, internamente coherente y ambientalmente destructivo, está tan arraigado y tan completamente integrado que parece inmune a cualquier esfuerzo por cambiarlo. Sin embargo, dado que también es tan complejo e interconectado, hay un número 
aparentemente infinito de lugares en los que se puede entrar en el sistema y, con suerte, interrumpirlo con fines positivos.

Con el objetivo de convertir la educación empresarial en un vehículo para transformar todo nuestro sistema global de producción-distribución-consumo, la solicitud IAJBS / CJBE para el concurso de la Fundación MacArthur 100\&change en 2016 fue solo una de las muchas posibilidades. El 2 de junio de 2016, la Fundación MacArthur anunció un concurso de 100 millones de dólares estadounidenses para resolver un problema social importante. La posibilidad de que las escuelas de negocios jesuitas participaran en el concurso se discutió brevemente durante la reunión de negocios del CJBE el 10 de julio en Le Moyne College en Syracuse, Nueva York. Una semana después, en el $23^{\circ}$ Foro Mundial de la IAJBS en Nairobi, Kenia, se aprobó la siguiente resolución por unanimidad-que fue aprobada al día siguiente por la Junta Ejecutiva de la IAJBS:

\begin{abstract}
La reunión anual de la IAJBS solicita a los líderes de la IAJBS, a la dirección del CJBE y al resto de la red de escuelas de negocios jesuitas que trabajen juntos para participar en el concurso de 100 millones de dólares llamado 100\&change de la Fundación MacArthur con un proyecto para transformar la educación empresarial jesuita para que esté completamente alineada con la sabiduría de Laudato Si', con nuestros principios educativos jesuitas universalmente válidos, y con la necesidad de la sostenibilidad global, la justicia social y la lucha contra la pobreza. (18 de julio 2016)
\end{abstract}

El 2 de octubre de 2016 se presentó a la Fundación MacArthur una propuesta para utilizar la transformación de la educación jesuita y de toda la educación en management como un vehículo para transformar nuestro sistema global de producción-distribución-consumo. Hay un poco de ambigüedad con respecto a cómo se cuentan las distintas solicitudes, pero en una cuenta el número es 1,407. Según este recuento, la de IAJBS / CJBE fue una de las 1.406 solicitudes que no ganó el premio de 100 millones de dólares.

La fecha límite para la presentación de candidaturas al concurso 100\&change de 2019 finaliza en agosto de 2019. Actualmente se están realizando esfuerzos para redactar una nueva solicitud que sea muy parecida a la original de 2016. Esta nueva solicitud seguirá invitando a las escuelas jesuitas y otras escuelas de negocios a transformar sus currículos y gran parte de su investigación para alinearlos con las realidades del siglo XXI y las necesidades de un mundo regenerativo. De hecho, la posibilidad de tal solicitud ya se ha descrito en esta revista (Stoner, 2018). 
No sería necesario que un miembro del claustro de profesores estuviera en una universidad que gane el premio de la Fundación MacArthur, un evento altamente improbable, para contribuir a la transformación de la educación empresarial y nuestro sistema global de producción, distribución y consumo. Cualquier profesor en cualquiera de las disciplinas de una escuela de negocios puede revisar su programa de estudios con el fin de decidir qué es apropiado para las realidades del siglo XX en comparación con lo que es apropiado para las del XXI, y luego comenzar a hacer ajustes en la manera de enseñar e investigar que reclama las realidades del siglo XXI. De hecho, el profesorado de la Facultad de Negocios Anderson de la Universidad Regis y de la Escuela de Negocios Gabelli de Fordham están comenzando o han estado ocupados en este tipo de investigación. Dado que es difícil justificar la enseñanza de un programa de estudios que es apropiado para el siglo XX y no para el XXI, es muy probable que muchos otros sigan su ejemplo explorando por su cuenta y compartiendo lo que están haciendo y aprendiendo con los demás.

\section{¿CÓMO MARCAR UNA DIFERENCIA? CASI DEMASIADAS OPORTUNIDADES PARA ELEGIR}

A medida que se escribía este editorial, el Anderson College of Business anunció una nueva especialización en su programa de Máster en Ciencias en Finanzas y Economía, en colaboración con el Instituto Capital (Capital Institute) y otros socios comprometidos en explorar y crear enfoques de finanzas regenerativas a nivel local y global. El programa abordará exactamente los problemas en el sistema financiero global que son un obstáculo para la creación de un mundo sostenible / floreciente / regenerador.

Hay muchas oportunidades para cada uno de nosotros en lo que enseñamos, lo que investigamos, cómo definimos el servicio, lo que elegimos comprar o alquilar, y consumir, en qué invertimos y cómo votamos. Las preguntas difíciles no se refieren a la búsqueda de oportunidades y desafíos, sino a la posibilidad de elegir entre muchas alternativas atractivas. Y sobre cuánto de nuestro tiempo, energía y otros recursos dedicaremos a las selecciones que hacemos.

Sabemos cuál es el problema. La pregunta es lo que haremos cada uno de nosotros al respecto. 


\section{Y AHORA HABLEMOS DE LOS ARTÍCULOS EN ESTE NÚMERO DE LA REVISTA}

Cada uno de los cinco artículos en este número proporciona maneras de que el cambio y la transformación a nivel personal y / o de sistemas pueden contribuir a un mundo más sostenible.

Bernard Arogyaswamy argumenta que, si bien la innovación a menudo crea una ventaja competitiva y un crecimiento económico, también puede tener impactos negativos: por ejemplo, el uso recursos ya limitados, los daños ambientales, las desigualdades sociales e incluso una movilidad social disminuida. Al describir cómo se pueden diseñar y elegir las estrategias de innovación para contribuir de manera más efectiva a la creación de un mundo sostenible y cómo las acciones centradas en la sostenibilidad pueden ser una fuente de innovación, desarrolla una matriz de tres por cuatro que ofrece un marco para crear y analizar iniciativas e ideas centradas en la sostenibilidad. Coloca la innovación de producto, proceso y gestión en un eje y cuatro enfoques de sostenibilidad (reducción de costos y acciones enfocadas en la diferenciación para la sostenibilidad ambiental; acciones dirigidas por los empleados y la comunidad para la sostenibilidad social) en el otro.

El resultado es una serie de 12 estrategias de sostenibilidad que las empresas pueden usar como guías para lograr objetivos como la reducción de emisiones, menos desperdicio de material y mayor bienestar de los empleados y de la comunidad, entre otros.

Para ayudar en el cambio hacia la energía renovable, Claire Siegrist y Evangelos Katsamakas presentan los resultados de un proyecto de investigación de educación empresarial que analizó la cuestión de la generación de electricidad utilizando un sistema distribuido basado en energía renovable frente a uno centralizado basado en fósiles combustibles. Describen un sistema de apoyo a las decisiones que puede ayudar a los responsables políticos y las partes interesadas a evaluar la viabilidad de los sistemas de energía solar para los tejados. El sistema utiliza medidas basadas en modelos de evaluación regionales existentes y que incluyen información sobre variables como los costes para los consumidores, la demanda regional y el apoyo del gobierno. El documento muestra cómo calcular los costes y las cantidades de electricidad generada para ver cómo un sistema de energía renovable podría funcionar contra los combustibles fósiles tradicionales y cómo podría reducir las 
emisiones en general. Por lo tanto, el uso del sistema de soporte puede llevar a acciones que ayudarán a reducir los costes y las emisiones, incluso si la generación distribuida no puede reemplazar completamente los sistemas centralizados hasta el momento.

Otra herramienta que es el resultado de un proyecto de educación empresarial proviene del trabajo de Karyl Leggio y el Coronel Reid Nichols. Los estudiantes usaron la simulación de Monte Carlo (una técnica que a menudo se usa para medir el riesgo) como un dispositivo de modelización financiera para apoyar las decisiones sobre cómo asignar recursos y justificar los costes relacionados con el Sistema de Boyas Interpretativas de la Bahía de Chesapeake, una red de boyas que proporciona a los usuarios la información técnica y científica necesaria para "mejorar los pronósticos marinos" y "monitorear la salud de la Bahía [de Chesapeake]." La herramienta resultante manejó la complejidad suficiente y tenía la sustancia suficiente para ser utilizada por la Administración Nacional Oceánica y Atmosférica (National Oceanic and Atmospheric Administration o NOAA) en su solicitud de presupuesto al Congreso. Se consideró particularmente útil para ayudar a la NOAA en su tarea de proteger y preservar la Bahía de Chesapeake, una importante fuente de mariscos y la base de un puerto importante en la costa este de los Estados Unidos.

El trabajo de Quan Le y Grace Jovanovic resalta la importancia de las colaboraciones para transformar vidas individuales y mover los sistemas comerciales hacia la obtención de resultados más sostenibles. Utilizando un modelo comercial en el que el café se compra directamente a los agricultores nicaragüenses a precios justos que respetan los precios mínimos previamente establecidos, el Café Ambiental, creado por los estudiantes, ofrece a sus productores de café estabilidad económica y estímulo. Esto, a su vez, permite a los agricultores hacer la transición a la agricultura orgánica al mismo tiempo que mejora la salud, la educación y el bienestar económico de sus familias. Los estudiantes y profesores que trabajan en esta colaboración con los agricultores, por otro lado, experimentan aspectos de transformación personal a través de negocios y lecciones de vida que son consistentes con el objetivo de solidaridad con los marginados.

Alain Decrop y Antje Graul, reconociendo las muchas formas en que el progreso en la economía compartida puede contribuir a un mundo más sostenible, abordan el desafío de mejorar la participación de los proveedores en dicha economía a través 
de esquemas de consumo colaborativos que pueden generar menos desperdicio, reducción de nuevas compras, así como una mayor recirculación de productos. Su estudio presenta evidencia de que tanto la percepción reducida del riesgo como la mejor confianza en el sistema pueden mejorar la probabilidad de participación en una plataforma de intercambio. Los consumidores están más dispuestos a compartir sus activos como proveedores en lo que los autores denominan un acuerdo de "compensación recíproca (monetaria)" que en una configuración de "reciprocidad generalizada" porque perciben un mayor grado de riesgo con este último—no tienen garantía de "lo que están recibiendo a cambio" en la situación de reciprocidad generalizada. Los autores también muestran que estos esquemas de consumo colaborativo son más atractivos cuando un intermediario de mercado sin fines de lucro facilita el proceso de intercambio. Por lo tanto, es importante comprender estos aspectos de la economía del intercambio como tal y tomar medidas sobre ellos si la oferta de activos compartidos es crecer, satisfacer la creciente demanda de recursos compartidos y lograr las ventajas de hacerlo.

Ciertamente, como lo sugieren estos proyectos inspirados en la educación en management, es probable que haya muchos otros ejemplos en nuestras escuelas de negocios que puedan ayudarnos a crear un mundo más sostenible, floreciente y regenerador: ya sea porque nos ayuden a tomar decisiones a nivel de organización y de sistemas o porque nos inspiran a nivel personal.

\section{POSDATA}

Para aquellos de nosotros, por cierto, que estamos tentados a hacer el tipo de preguntas que hizo Hamming, notemos que concluyó su descripción de esos almuerzos de Bell Labs con la siguiente frase:

"No fui bienvenido después de eso ¡Tuve que encontrar a alguien con quien comer!" 


\section{BIBLIOGRAFÍA}

Collins, J. 2001. Good to great: Why some companies make the leap ... and others don't. Nueva York: Harper Business.

Diamond, J. 1999. Guns, germs, and steel: The fates of human societies. Nueva York: Norton.

Diamond, J. 2005. Collapse: How societies choose to fail or succeed. Nueva York: Viking/Penguin.

Diamond, J. 2019. How it ends: The fate of the planet hangs in the balance. How do we avoid the worst-case scenario? The New York Times Sunday Book Review, 21 de abril: 13 .

Ehrenfeld, J. 2009. Sustainability by design: A subversive strategy for transforming our consumer culture. New Haven, CT: Yale University Press.

Francis. 2015. Laudato si': On care for our common home. Ciudad del Vaticano: Libreria Editrice Vaticana.

Hamming, R. 1986. You and your research. Transcripción del Seminario del Coloquio de investigación de Bell Communications (7 de marzo). Disponible en https://www.cs.virginia.edu/ robins/YouAndYourResearch.html (Fecha de acceso 23 de abril, 2019).

Hawken, P. (Ed.). 2018. Drawdown: The most comprehensive plan ever proposed to reverse global warming. Nueva York: Penguin.

Krugman, P. 2016. Wind, sun and fire. The New York Times, 1 de febrero. Disponible en https://www.nytimes.com/2016/02/01/opinion/wind-sun-and-fire.html (Fecha de acceso 22 de abril, 2019).

Lanchester, J. 2019. World on fire. The New York Times Sunday Book Review, 28 de abril: $1,20$.

Laszlo, C., Sroufe, R., \& Waddock, S. 2017. Torn between two paradigms: A struggle for the soul of business schools. AI Practitioner, 19(2): 108-119. 
Lovins, L. H., Wallis, S., Wijkman, A., \& Fullerton, J. 2018. A finer future: Creating an economy in service to life. Gabriola, BC: New Society Publishers.

Lowney, C. 2003. Heroic leadership: Best practices from a 450-year-old company that changed the world. Chicago: Loyola Press.

McKibben, B. 1989. The end of nature. Nueva York: Random House.

McKibben, B. 2010. Eaarth: Making a life on a tough new planet. Nueva York: Times Books/Henry Holt.

McKibben, B. 2019. Falter: Has the human game begun to play itself out? Nueva York: Henry Holt.

Pirson, M. 2017. Humanistic management: Protecting dignity and promoting wellbeing. Nueva York: Cambridge University Press.

Rich, N. 2019. Losing earth: A recent history. Nueva York: Farrar, Straus, \& Giroux.

Scharmer, O. 2016. Theory U: Leading from the future as it emerges: The social technology of presencing (2nd ed.). Oakland, CA: Berrett-Koehler.

Stoner, J. A. F. 2013. What we want this journal to be: Our first editorial essay in which we hope to start a continuing and evolving conversation about why we are now creating this new journal and what we want it to become. Journal of Management for Global Sustainability, 1: 1-6.

Stoner, J. A. F. 2018. Innovation in educational and societal transformation: The MacArthur Foundation, Jesuit business schools, and the world. Journal of Management for Global Sustainability, 6(2): 19-35.

Tsao, F. C., \& Laszlo, C. 2019. Quantum leadership: New consciousness in business. Ciudad de Redwood, CA: Stanford University Press.

Wallace-Wells, D. 2019. The uninhabitable earth: Life after warming. Nueva York: Tim Duggan Books/Crown Publishing.

Your dictionary. n.d. Technology. Disponible en https://www.yourdictionary.com/ technology (Fecha de acceso 23 de abril, 2019). 